\title{
Separating distractor rejection and target detection in posterior parietal cortex-An event-related fMRI study of visual marking
}

\author{
S. Pollmann, ${ }^{\mathrm{a}, \mathrm{b}, *}$ R. Weidner, ${ }^{\mathrm{a}}$ G.W. Humphreys, ${ }^{\mathrm{c}}$ C.N.L. Olivers, ${ }^{\mathrm{c}}$ K. Müller, ${ }^{\mathrm{b}}$ G. Lohmann, ${ }^{\mathrm{b}}$ \\ C.J. Wiggins, ${ }^{\mathrm{b}}$ and D.G. Watson ${ }^{\mathrm{d}}$ \\ ${ }^{a}$ Day Clinic of Cognitive Neurology, University of Leipzig, Leipzig, Germany \\ ${ }^{\mathrm{b}}$ Department of Neurology, Max-Planck-Institute of Cognitive Neuroscience, Leipzig, Germany \\ ${ }^{\mathrm{c}}$ Behavioural Brain Sciences Centre, School of Psychology, University of Birmingham, Birmingham, UK \\ ${ }^{\mathrm{d}}$ Department of Psychology, University of Warwick, Coventry, UK
}

Received 12 March 2002; revised 15 August 2002; accepted 21 October 2002

\begin{abstract}
Successful survival in a competitive world requires the employment of efficient procedures for selecting new in preference to old information. Recent behavioral studies have shown that efficient selection is dependent not only on properties of new stimuli but also on an intentional bias that we can introduce against old stimuli. Event-related analysis of functional magnetic resonance imaging data from a task involving visual search across time as well as space indicates that the superior parietal lobule is specifically involved in processes leading to the efficient segmentation of old from new items, whereas the temporoparietal junction area and the ascending limb of the right intraparietal sulcus are involved in the detection of salient new items and in response preparation. The study provides evidence for the functional segregration of brain regions within the posterior parietal lobe.

(C) 2003 Elsevier Science (USA). All rights reserved.
\end{abstract}

\section{Introduction}

In everyday life, new entrants to a scene appear against a background of old objects. Given the informational value of new items, how do we attend to new stimuli in preference to old? One possibility is that there is an automatic bias to favor new items (Theeuwes, 1994; Yantis, 1996); new events capture attention in an automatic manner. A second possibility is that new items are favored because there is passive decay of information within the attentional system; objects simply lose their "attentional salience" over time. A third possibility is that observers bring to bear active processes that bias processing against the old and so in favor of the new. Behavioral evidence for an active process of attentional bias comes from studies of a visual search across time as well as space. Watson and Humphreys (1997)

\footnotetext{
* Corresponding author: University of Leipzig, Day Clinic of Cognitive Neurology, Liebigstrasse 22a, D-04103 Leipzig, Germany. Fax: ++49-341-9724269.

E-mail address: pollmann@cns.mpg.de (S. Pollmann).
}

adapted a serial search procedure by presenting half the distractors in one time step before the occurrence of a second set of items (the distractors plus the target for search, when present; the "preview" search procedure). Although the old distractors from display 1 remained when the new stimuli appeared in display 2, search was as efficient as when only the second set of items was presented. There was no impact of the old items on search for the new. This was not a passive effect. If subjects engaged in a secondary task when the old items were first presented (shadowing a string of numbers), there was an impact of the old items on search for the new (Humphreys et al., in press; Watson and Humphreys, 1997). In order to keep old items out of search for the new, some attention needs to be paid to the old items in the first place. Humphreys et al. propose that old items are first encoded into a memory representation that is subsequently inhibited; a process termed visual marking.

Evidence for inhibition of old items comes from studies using a probe detection procedure (Olivers and Humphreys, 2002; Watson and Humphreys, 2000). Probes presented at the locations of old stimuli are difficult to detect, when 
subjects are set to search for new events (with probe events occurring on a minority of trials). However, differences in probe detection on old and new items are largely eliminated when probe detection is the main task and it is not embedded as part of the search task, even when the temporal intervals between the old and new stimuli are maintained. This is consistent with subjects adopting an intentional bias against old items, to facilitate selection of the new. The generality of this intentional bias in search has been shown by other studies in which marking has been shown with moving items (Watson and Humphreys, 1998) and even when a difficult search task is undertaken on the second set of items (Theeuwes et al., 1998).

In the present study we use event-related fMRI procedures to investigate the neural mechanisms of visual marking. To do this, it is important to isolate activation stemming from the processing of "old" items, under preview search conditions, from activation stemming from the subsequent search and target-detection process (conducted following display 2). We attempted to separate these activations using two different procedures. In Experiment 1 we analyzed differences in the time course of the blood oxygenation level-dependent (BOLD) response under different search conditions. Early onset of the BOLD response in the preview condition, relative to appropriate baselines, can be indicative of visual marking of items in display 1. In Experiment 2 , we again measured activation across different search conditions, but this time included a proportion of "preview only" trials, where the BOLD response should not be affected by activation linked to search displays (display 2). Here contrasts in the magnitude of the BOLD response can be informative of visual marking applied to items in display 1 .

In both experiments, subjects had to search for a predetermined target stimulus, a blue $\mathrm{H}$, within the second of two consecutively presented visual displays (each presented for $2 \mathrm{~s}$; Fig. 1). In one condition ("single-feature search"), the blue $\mathrm{H}$ target was presented along with blue As as distractor items. Here the target differed by a single feature (form) from the distractors. In a more difficult, serial search condition ("conjunction search"), the blue $\mathrm{H}$ was presented along with green $\mathrm{H}$ and blue A distractors. In such a condition, when the target is defined only by a conjunction of features (form and color), search is generally less efficient and characterized by linear search functions (Treisman and Gelade, 1980). In a third, preview condition, the second display was identical to the conjunction search condition. However, one set of distractors (the green Hs) was presented in advance in the first display, providing observers with early information about which distractors to ignore. To control for effects of physical stimulus presentation, the search displays in the single-feature and conjunction search conditions were preceded by a set of red Os ("dummy previews"), which occupied the same locations as the green $\mathrm{H}$ distractors in the preview condition but which differed by their color and shape from the stimuli in the actual search display. The red Os should have little impact on search and so should not be actively ignored, unlike the green $\mathrm{Hs}$ in the preview condition. ${ }^{1}$

Prior behavioral work shows that search in the singlefeature and preview conditions can be equally efficient, and, in both conditions, search is more efficient than in the conjunction search treatment (Watson and Humphreys, 1997). Thus contrasts between the single-feature and preview conditions, on the one hand, and the conjunction condition, on the other, should inform us about processes sensitive to the ease of search or postsearch decision and response preparation. Uniquely in the preview condition, the efficient search may also be dependent on visual marking of the old stimuli. Contrasts between the preview condition, on the one hand, and the single feature and conjunction conditions, on the other, should thus be informative about the processes involved in visual marking. In both single-feature and conjunction baselines, subjects are unlikely to engage in active ignoring of the completely irrelevant dummy previews (the red Os).

Experiment 1 concentrated on analyzing the temporal differences in the BOLD response in the three critical search conditions, differentiating brain regions in which (a) there were temporal changes unique to the preview condition (linked to active ignoring of old items), and (b) there were temporal changes common to the single-feature and preview condition, when compared with the conjunction baseline (linked to target detection and/or decision and response preparation). Early onset of the BOLD response in the preview condition can be taken as diagnostic of processes involved in actively ignoring the old stimuli. A delayed onset in the conjunction baseline, relative to single-feature and preview search, will be diagnostic of decision and response preparation processes emerging more rapidly (because search is completed more quickly) in the latter conditions. In Experiment 2 we attempted to derive a direct measure of activation associated with actively ignoring old items in search, this time contrasting the magnitude of activation associated with processing stimuli on previewonly trials $(1 / 3$ of the total number of trials). These trials were included in order to enable us to measure activation associated with the preview displays uncontaminated by activation associated with search displays. We also used three stimulus onset asynchronies (SOAs) between the preview and the search displays (SOAs presented randomly), and we randomly mixed the search conditions. These extra changes were incorporated to equate potential differences in adopting a strategic set across the critical conditions.

\footnotetext{
${ }^{1}$ Our prior behavioral studies have demonstrated little impact of such red Os on search in equivalent single feature and conjunction searches to those used here.
} 
a Single feature

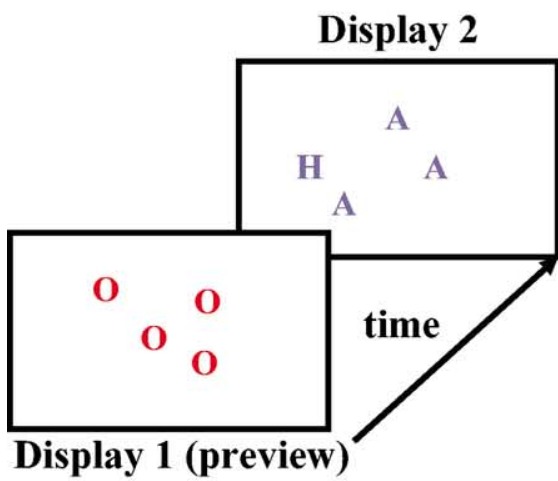

Single feature

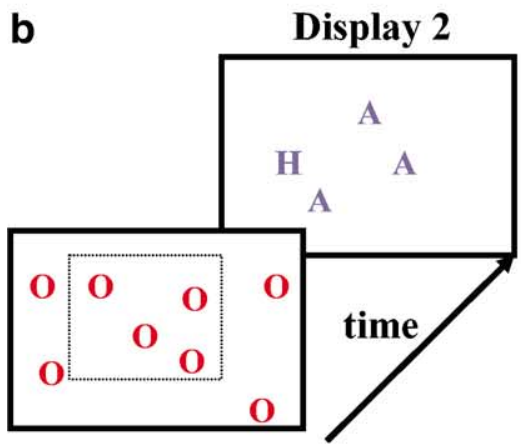

Display 1 (preview)
Conjunction

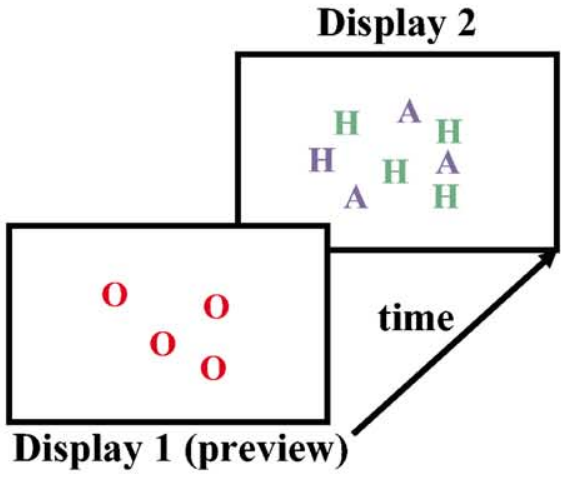

Conjunction

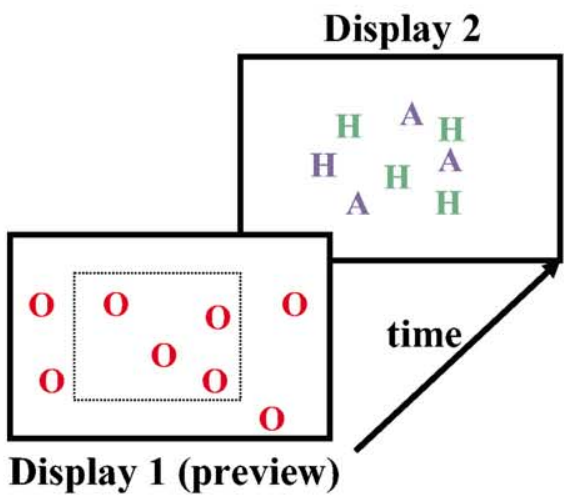

Preview
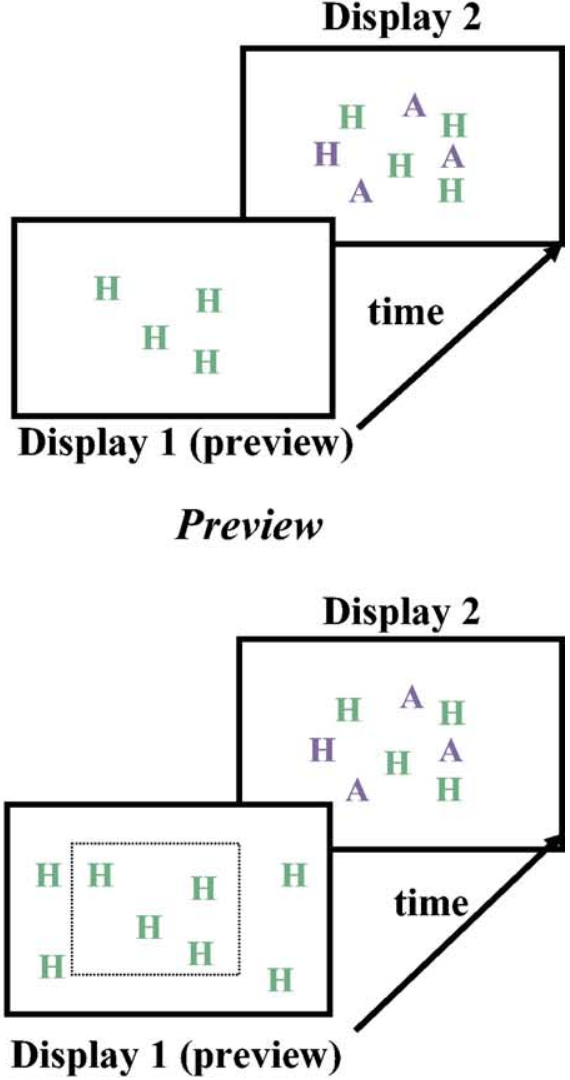

\section{Dummy preview only}

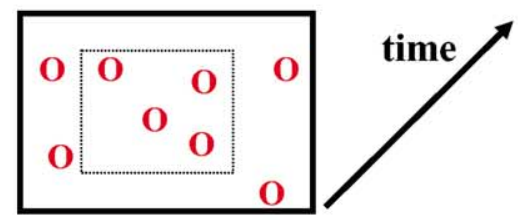

Preview only

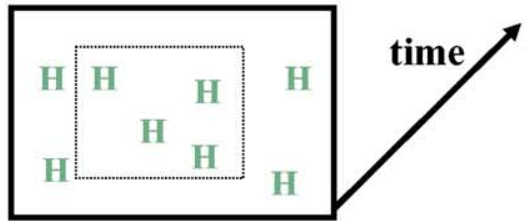

Fig. 1. (a) Examples of the displays presented in Experiment 1. In the single-feature and conjunction baseline conditions, the previews were red Os; in the preview condition they were green Hs. The target was always a blue $\mathrm{H}$ presented in display 2 (the search display). (b) In Experiment 2, the first display contained a region of extra items (cf. Experiment 1), which, in each condition, disappeared when the second search display was presented. This was done to emphasize that old items were irrelevant to subsequent search. For illustrative purposes only, a dotted rectangle is drawn separating the central items in the previews from the peripheral items. This was not present in the actual study. There was also an extra preview-only condition in which no search displays appeared and only previews were presented. For both Experiments 1 and 2, we illustrate only a display size of 8 items (in the final conjunction display; 4 items in the single-feature search condition).

\section{Methods}

\section{Subjects}

Eight subjects took part in the first fMRI experiment, five of them female. Subjects' ages ranged between 22 and 26 years, with a mean age of 23 years. In the second fMRI experiment there were 10 subjects ( 5 male $/ 5$ female) whose ages ranged between 22 and 27 years, with a mean age of 24.8 years. All subjects were right-handed, assessed by means of the Edinburgh Inventory (Oldfield, 1971). Each subject gave prior informed consent according to the Max Planck Institute guidelines. The fMRI procedures were approved by the local ethics review board at the University of Leipzig.

\section{Experimental design}

Stimuli were projected by an LCD projector on a backprojection screen mounted in the bore of the magnet behind the subjects' head. Subjects viewed the screen wearing 
mirror glasses, which were equipped with corrective lenses if necessary. Each trial began with the presentation of a white fixation cross for $6 \mathrm{~s}$, followed by two consecutive stimulus displays, each presented for $2 \mathrm{~s}$. In a previous paper, we have shown that an interstimulus interval (ISI) of $6 \mathrm{~s}$ leads to a reduction of the BOLD effect size (signal change/error variance) due to overlap of successive BOLD responses of only $\sim 10 \%$, whereas further ISI reduction led to a sharp decrease (Pollmann et al., 1998). Therefore, an ISI of $6 \mathrm{~s}$ was chosen to optimize statistical power (i.e., maximal number of repetitions/minimal reduction in effect size). Stimuli in Experiment 1 were colored letters which were randomly distributed over an area of $5.2^{\circ} \times 5.2^{\circ}$ (Fig. 1a). In Experiment 2, the conditions were randomized, which could act against subjects adopting a strategic set to ignore old items in the preview condition. To counteract this, we sought to maximize the perception of "dummy" preview displays as containing background, irrelevant items (akin to wallpaper). To do this, we presented stimuli across a larger spatial area $\left(17.3 \times 17.3^{\circ}\right)$, with extra items being added to the borders of the preview display as a function of the number of items in the central $5.2 \times 5.2^{\circ}$ area. When display 2 occurred, the items in the central area either changed (in the single-feature and conjunction conditions) or remained the same (in the preview condition); the items in the outer area offset (in all conditions) (see Fig. 1b). The density of items in the border regions matched that in the central regions of the previews. The positions of the items on preview trials always matched the positions of the green $\mathrm{H}$ distractors (when they occurred) in display 2. In the conjunction condition this meant that half the new items occupied the locations of the dummy red Os. In the singlefeature condition, the new items occupied locations different than those taken by red dummy Os (since no green distractors appeared). In prior behavioral studies we have used similar search conditions and found little effect of these dummy previews on standard single-feature and conjunction searches. The slopes for these conditions in the behavioral data we report here are also comparable to those obtained in previous reports (Watson and Humphreys, 1998). In the single-feature search condition, stimulus presentation began with red Os (display 1), followed by the presentation of blue A distractors and the blue $\mathrm{H}$ target (display 2). In the conjunction search condition the stimuli presented were red Os (display 1) which were followed by the target plus blue $\mathrm{A}$ and green $\mathrm{H}$ distractors (display 2). In the preview condition, stimulus presentation began with the green $\mathrm{H}$ distractors (display 1 ) which were followed by the blue A distractors and the blue $\mathrm{H}$ target. Examples of the display sequences are shown in Fig. 1a and $1 b$.

The display size was 8 or 12 items in Experiment 1 and 8 or 16 in Experiment 2, on respectively $50 \%$ of the trials each. The order of the different display sizes was randomly determined. The task was to decide whether the target (the blue $\mathrm{H}$ ) fell to the left or right of fixation. In Experiment 1 the conditions were blocked and there was a single SOA of
$2 \mathrm{~s}$ between the onset of display 1 and that of display 2. In Experiment 2, the search conditions were not blocked but occurred randomly, and on one-third of the trials only preview displays were presented (display 1 only, containing one set of distractors or red "O" dummies in 50\% of trials, followed by a blank field instead of display 2). There were also three SOAs between the onset of display 1 and display 2 (1666, 2000, and $2333 \mathrm{~ms}$ ), which occurred equally often (but in a random order) across the trials.

\section{fMRI methods}

A session of Experiment 1 consisted of 4 scans, one scan for each experimental condition. A scan started with the presentation of a fixation cross for $30 \mathrm{~s}$, followed by 32 trials and ended with a 30-s fixation period. The order of conditions was balanced across subjects. On the day before the fMRI experiment, subjects took part in a training session consisting of the same experiment which was run in the scanner.

The fMRI data were acquired at $3 \mathrm{~T}$ by a Bruker $30 / 100$ Medspec system (Bruker Medizintechnik, Ettlingen, Germany) using a gradient-recalled EPI sequence which included bipolar gradient pulses to induce very mild diffusion weighting ( $b$ value 20 ) in order to suppress signal from flowing blood. The flip angle was $55^{\circ}, \mathrm{TR}=666 \mathrm{~ms}$, with a TE of $42 \mathrm{~ms}$. The matrix size was $64 \times 64$, FOV $19.2 \mathrm{~cm}$, with $100-\mathrm{kHz}$ sampling. In the first experiment, five axial slices were acquired with $8-\mathrm{mm}$ slice thickness and 2-mm interslice gap. The slices were oriented horizontally with the most ventral slice in the plane through the anterior and posterior commissures (AC-PC).

In the second experiment we were able to measure 9 slices at TR $=666 \mathrm{~ms}$, due to hardware improvements. They were aligned with AC-PC, with the second-most ventral slice in the AC-PC plane. Subjects undertook 4 blocks (acquired within a single scan) with the conditions presented at random within each block.

Previous work has shown that shifts of BOLD onset of less than a second can be detected between experimental conditions (Dymond et al., 1999; Menon et al., 1998). The short repetition time applied in the present study allowed us to sample the BOLD response three times (in Experiment 1, and between 2 and 4 times in Experiment 2) during the presentation of each display, thus securing a sound database for the analysis of small temporal differences between experimental conditions.

The data were analyzed with the software package LIPSIA (Leipzig Image Processing and Statistical Inference Algorithms; Lohmann et al., 2001). First, slice acquisition time differences were corrected by sinc interpolation. Then, data were corrected for movement artefacts following the algorithm of Friston et al., (1996). Baseline drifts were corrected by high-pass filtering, implemented using a discrete Fourier transform. In the spatial domain, the data were filtered with a Gaussian filter with FWHM $=7 \mathrm{~mm}$. Fol- 
lowing this preprocessing, the functional datasets were coregistered with the subjects' individual highresolution anatomical datasets and normalized by linear scaling. This process included a resampling of the data using trilinear interpolation. After resampling the data had a resolution of $3 \times 3 \times 3 \mathrm{~mm}$. Data were analyzed using the general linear model as laid down in Friston et al., (1995). In order to obtain volumetric measurements of activation areas we increased the spatial resolution of the SPM to $1 \times 1 \times 1 \mathrm{~mm}$ using trilinear interpolation. The volume of the activation areas is based upon this resampled SPM.

For Experiment 1, event-related analyses were computed using a model of the hemodynamic response and its temporal derivative (Friston et al., 1995; Josephs et al., 1997). This procedure allows to testing of BOLD temporal onset differences separately for significance in areas without significant amplitude differences (Friston et al., 1998; Josephs and Henson, 1999). Temporal onset differences were only analyzed in areas without significant amplitude differences. The significance criterion was $\alpha=0.001$, uncorrected for multiple comparisons. A more complex model, allowing for a separate test of differences in the dispersion of the BOLD response (via the second temporal derivative), was used in a probatory analysis. Because no significant differences of the dispersion were observed, we used the simpler model described above. To assess the temporal onset of activations, contrasts between search conditions were calculated individually for temporal onset differences. The group activations for the between-condition contrasts were calculated by one-sample $t$ tests at corresponding voxels of the individual $\operatorname{SPM}\{z\}$ across subjects (Bosch, 2000). The marking pattern was defined as a significantly earlier onset in the preview condition, compared to a single-feature search and conjunction search, separately, and no significant onset difference between single-feature search and conjunction search. The postsearch pattern was defined as a significant lag of the conjunction search onset relative to the single-feature search and preview conditions, respectively, with no significant onset difference between single-feature search and preview condition.

In Experiment 2, a simple model of the hemodynamic response was applied (Friston et al., 1995; Josephs et al., 1997), with fixed temporal parameters, in order to optimize detection of display-specific BOLD responses. Activation elicited by visual marking in the preview condition was assessed by comparing the preview-only condition (in which the preview display was presented without subsequent search display) to the dummy-only condition (in which the noninformative dummy display was presented without subsequent search display). Activation generated by visual search in display 2 was assessed after subtracting the preview-only condition from the preview condition, and the dummy-only condition from the single feature and conjunction search conditions. The significance criterion in Experiment 2 was set to a conservative $\alpha=0.0001$ (uncorrected) to yield a stringent confirmation of the results of Experiment
1. Blood oxygenation level-dependent signal time courses were extracted from the preprocessed individual datasets at the location of the group maximum. We calculated repeated-measures analyses of variance (ANOVAs) of search condition and time over the signal time courses to analyze amplitude and timing differences between conditions. For the assessment of amplitude changes, the main effect of condition was calculated in a time window of 24 images $(=$ $16 \mathrm{~s}$ ) after onset of the first display. Temporal differences in the development of the BOLD signal between conditions were analyzed by the interaction of search condition $\times$ time over the same time window. Additionally, we analyzed the signal in a restricted time window of 6 images $(=4 \mathrm{~s})$ after onset of the first display (in the contrasts testing visual marking-related activation) or the second display (in the contrasts testing search-related activation). An early onset of the BOLD response should lead to increased signal strength in this time window, indicated by a main effect of condition.

\section{Results}

\section{Behavioral data}

The mean correct reaction times (RTs) are shown in Fig. 2 ( $2 \mathrm{a}$ for Experiment 1, and $2 \mathrm{~b}$ for Experiment 2). There were no errors. In Experiment 1, RTs differed significantly between conditions $(F(2,6)=6,68, P<0.05)$. Reaction times were longer in conjunction search than in either single-feature search or preview condition (both $P<0.05$, Scheffé test). Slopes of the RT-display size search functions were $4.8,26.8$, and $7.7 \mathrm{~ms} /$ item in the single-feature, conjunction, and preview displays. There were reliable effects of condition and display size $(F(2,6)=6.68$ and $F(1,7)=$ 6.64 , both $P<0.05)$. The condition $\times$ display size interaction did not reach significance $(F(2,6)=1.41, P>0.05)$, due to the relatively small trial and subject numbers used. Nevertheless, the slopes of the search functions indicate that search was relatively efficient in the single-feature and preview conditions (slopes $<10 \mathrm{~ms} /$ item) and inefficient in the conjunction condition (slope $>25 \mathrm{~ms} / \mathrm{item}$ ) (see Treisman and Gormican (1988) for discussion of how slope differences distinguish different search processes).

In Experiment 2 RTs again differed significantly between the search conditions $(F(2,18)=27.64, P<0.001)$. RTs were longer in conjunction search than in either singlefeature search or preview condition (both $P<0.01$, Scheffé test). Response latencies also varied with the display size $(F(1,9)=40.27, P<0.001)$. There was no effect of the stimulus-onset asynchrony (SOA) $(F<1.0)$, and no interactions involving this factor. There was however a reliable interaction between condition and display size $(F(2,18)=$ 5.05, $P<0.025)$. Slopes of the RT-display size function were 10,20 , and $15 \mathrm{~ms} /$ item in the single-feature, conjunction, and preview displays. There was a reliable difference 
a)

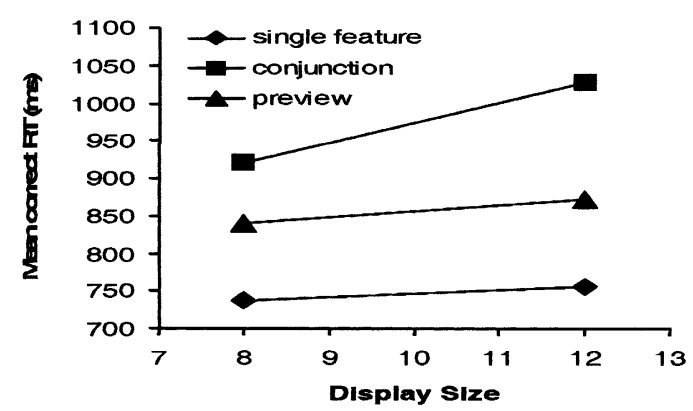

b)

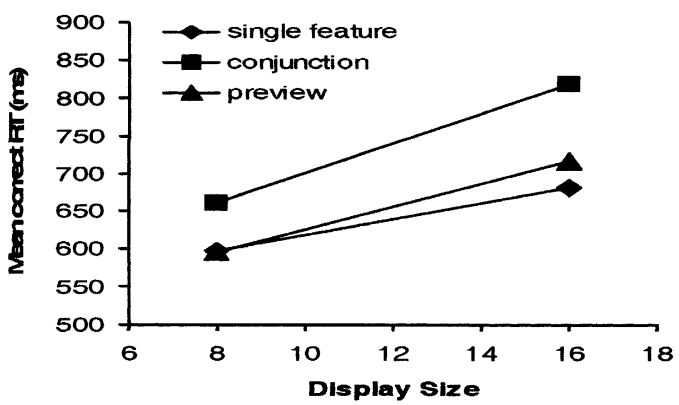

Fig. 2. (a) Mean reaction times (RTs) (ms) over subjects in the single-feature, preview, and conjunction search conditions of Experiment 1 as a function of the display size. (b) Mean RTs (ms) over subjects in the equivalent search conditions of Experiment 2.

in the slopes of the functions for the single-feature and conjunction conditions, indicated by a significant interaction between condition and display size when only these two conditions were compared $(F(1,9)=23.25, P<0.01)$. The slope of the function for the preview condition fell between that found in the single feature and conjunction conditions, and it did not differ reliably from either $(F(1,9)$ $=1.77$ and 2.2 , both $P>0.05$, for the condition $\times$ display size interactions for comparisons between the single-feature and preview and the conjunction and preview conditions). Judging from the search slopes, performance in the preview condition may have suffered in Experiment 2 because subjects were unable to optimize selection biases due to the randomized presentations of SOAs and search conditions. Nevertheless, absolute RTs were close in the preview and single-feature conditions. Experiment 1, in contrast, showed an intercept advantage for the single-feature baseline, presumably reflecting optimized expectancies for new displays when SOAs and conditions were blocked.

\section{Functional imaging}

\section{Experiment 1: temporal activation patterns}

To investigate which areas are involved in visual marking and visual search, respectively, we investigated temporal differences in the BOLD response in Experiment 1. Brain areas specifically involved in visual marking should show an earlier onset of the BOLD response in the preview condition compared to conjunction and single-feature search. This early onset reflects the opportunity to attentively process (and reject) the advance distractors in the preview condition.

Time-course differences may also arise because search was faster in the single-feature and preview search conditions, when compared with conjunction search. Therefore, brain areas involved in postsearch processes (target detection and response preparation) should show a lag in the BOLD onset for conjunction search versus single-feature search and preview conditions, but less difference between the single-feature search and preview conditions.

In the group averages for Experiment 1, the first pattern, indicative of visual marking (the "marking pattern") was found in the right posterior superior parietal lobule (SPL), bordering the precuneus (Fig. 3a, Table 1). The second pattern, indicative of postsearch processes (the "postsearch pattern") was found in several locations within the inferior parietal lobule. It was found on the lateral bank of the ascending limb of the right intraparietal sulcus (aIPS), and bilaterally in the temporo-parietal junction (TPJ) area, below the posterior segment of the superior temporal sulcus (pSTS). We extracted the BOLD signal at the locations of the highest $z$ values in the above analyses, to investigate the nature of the observed effects further.

Significant overall differences in signal amplitude between conditions were not observed in any of these areas (Table 2), ruling out that the observed time shifts were an artefact of different rise times for low and high amplitude signal changes. Significant main effects of time, indicating any change of the BOLD response in the time window, were observed in all areas with significant activations in the SPMs (and will therefore not be reported further, we refer to Table 2). Significant interactions of condition (preview, single-feature, and conjunction search) $\times$ time were observed in right SPL, aIPS, and pSTS, with a trend toward a significant interaction in left pSTS. These analyses confirmed the existence of condition-specific temporal shifts of the BOLD response in the areas which were significant in the statistical parametric map (SPM) based on the temporal derivative. However, the SPM analysis does not allow us to differentiate between onset shifts and peak shifts, but gives only an estimate of the integral shift of the BOLD response over time (Henson et al., 2002). For the differentiation of processes related to visual marking (starting with display 1) and visual search (starting with display 2), it is essential that the observed temporal differences in the search conditions reflect shifts in the onset of the BOLD response, associated 


\section{Early Onset in Preview Search}

\section{Late Onset in Conjunction Search}

a)
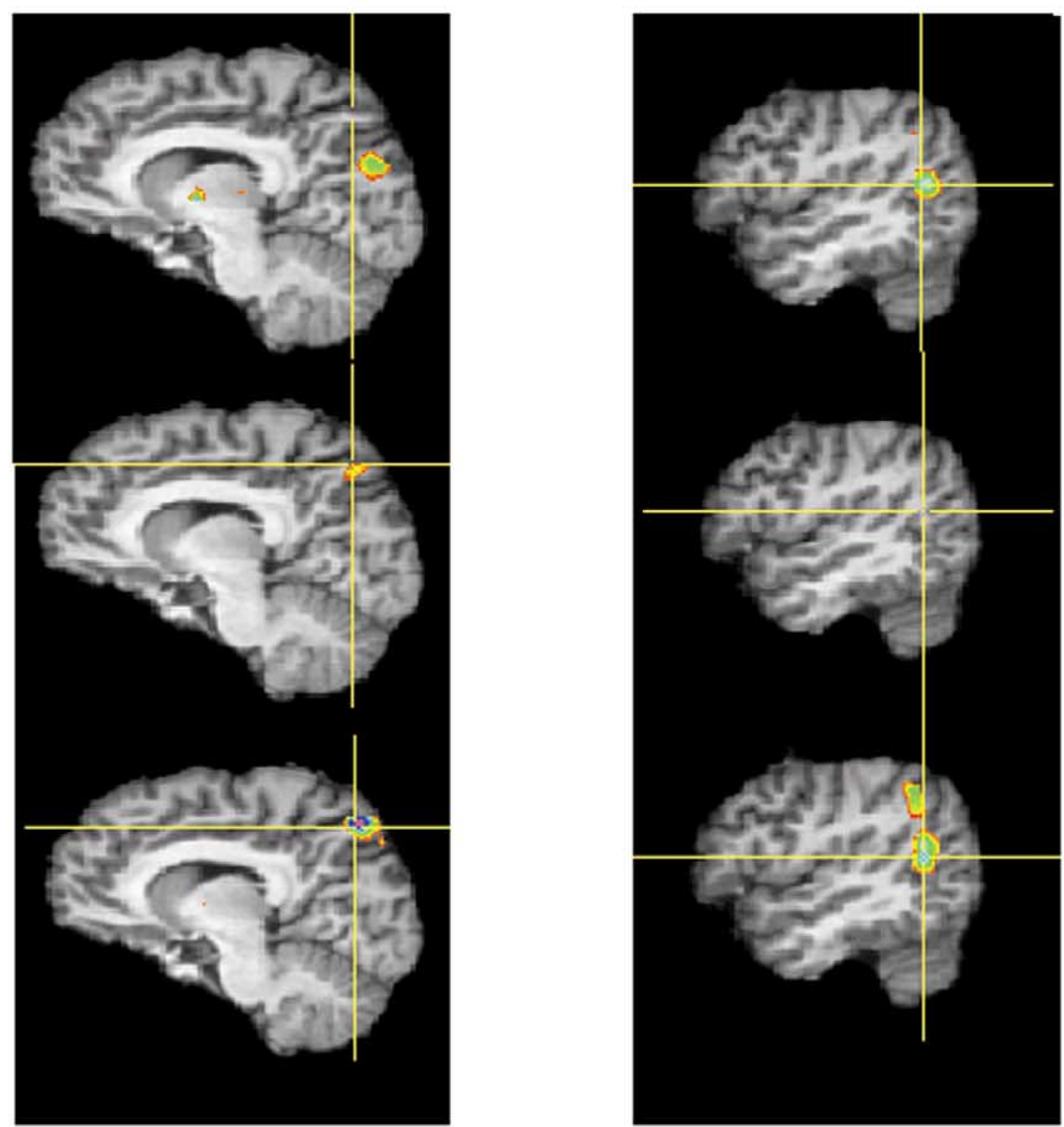

\section{$3.1 \square$}

\section{High Activation in \\ High Activation in Preview Condition Single Feature Search}

b)
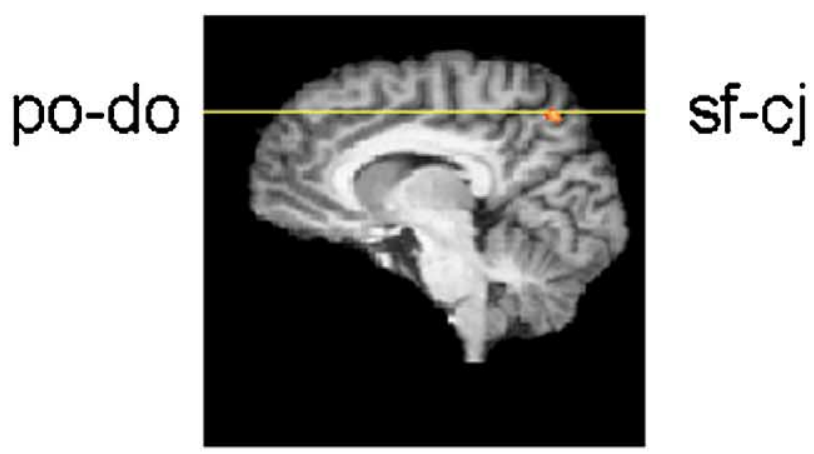

3.1

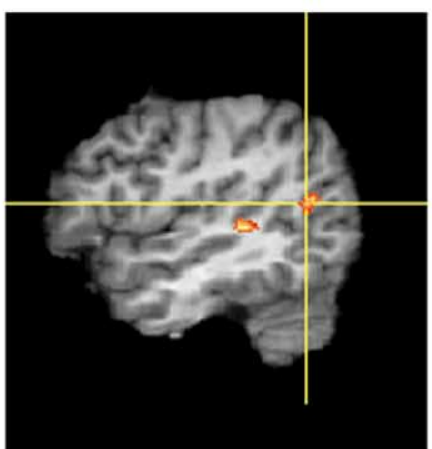

5

Fig. 3. (a) Experiment 1. Left column: Temporal group activation pattern related to visual marking in right superior parietal lobule $(x=7)$. Right column: Temporal group activation pattern related to post search processes in right posterior superior temporal sulcus $(x=43)$. The color scale represents temporal onset differences between single-feature search (sf)-conjunction search (cj), preview search (pv)-single feature search, and preview search-conjunction search. The scale indicates $z$ values. Positive values represent earlier onset of activation in the first condition of a contrast. (b) Experiment 2: (left column) Preview-related activation pattern in left SPL/precuneus $(x=7)$. (Right column) Significant signal increase in the single feature search-conjunction search contrast at the same location as the postsearch onset pattern in (a) $(x=43)$. $Z$ values represent differences in activation strength. Preview only (PO) indicates presentation of preview display without subsequent search display. Dummy only (DO) indicates presentation of dummy display without subsequent search display. 


\section{Experiment 1}
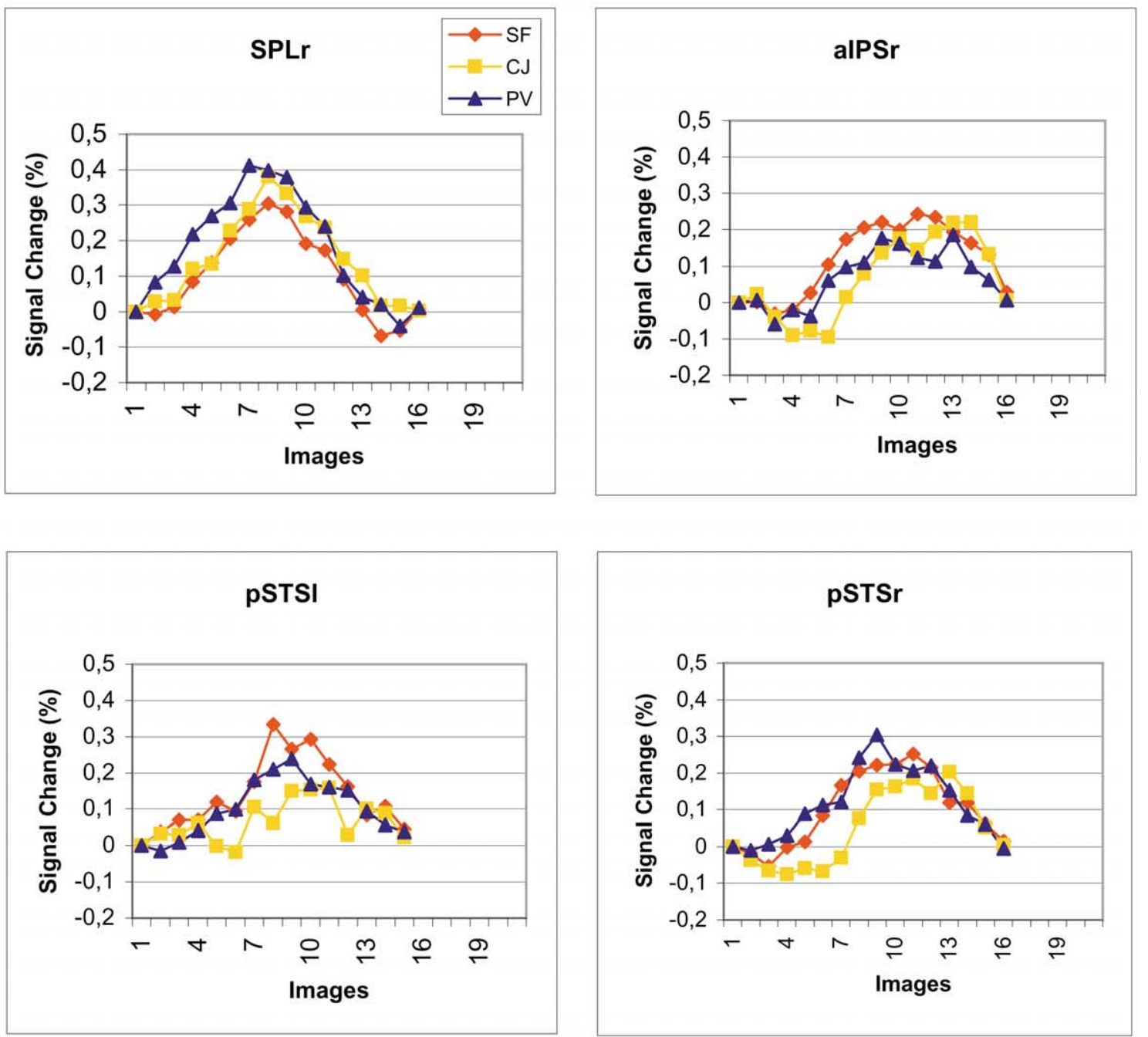

\section{Experiment 2}
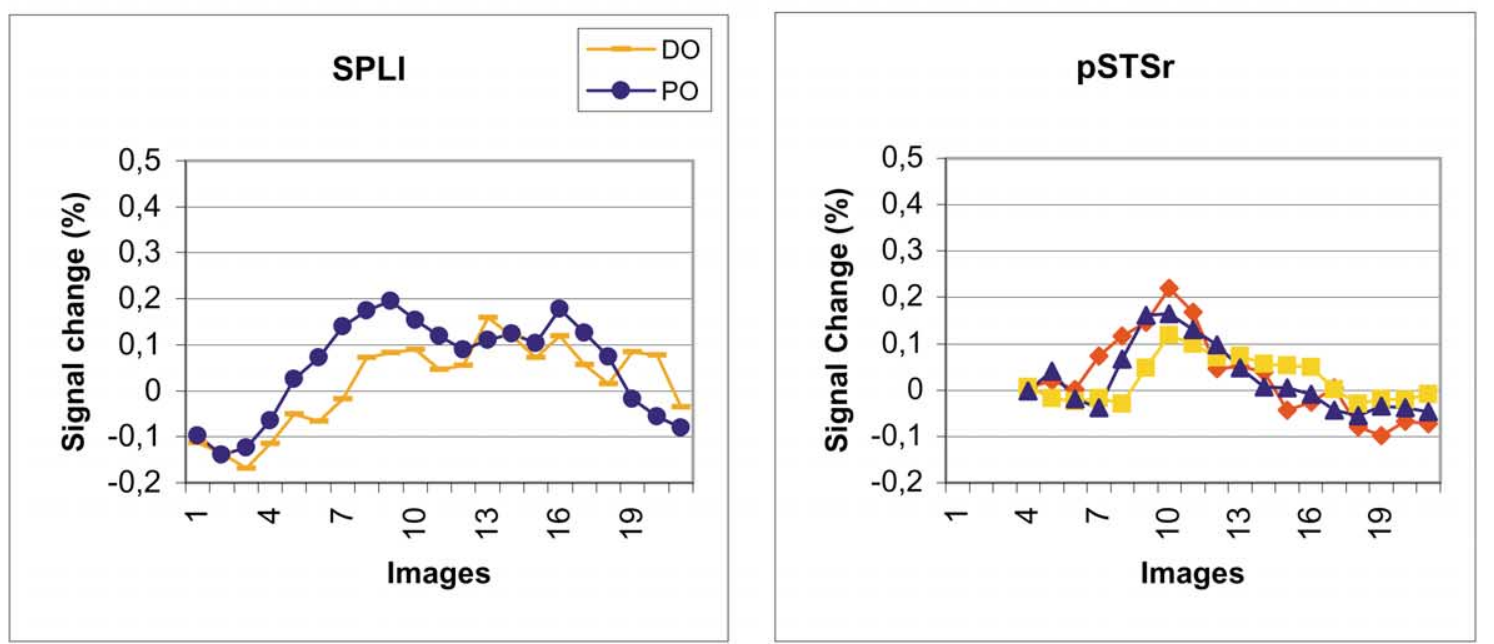

Fig. 4. BOLD time courses for areas which showed a significant marking- or postsearch-related activation pattern. The graphs show the averaged signal time course per condition. The $x$ axis denotes the number of images acquired at TR $=666 \mathrm{~ms}$ starting with the onset of the preview display. PV, preview search; CJ, conjunction search; SF, single-feature search; DO, dummy-only condition; PO, preview-only condition; SPL, superior parietal lobule; aIPS, anterior intraparietal sulcus; pSTS, posterior segment of the superior temporal sulcus; $-r$; right hemisphere; -1 left hemisphere. 
Table 1

Brain areas with activation changes related to visual marking or postsearch processes

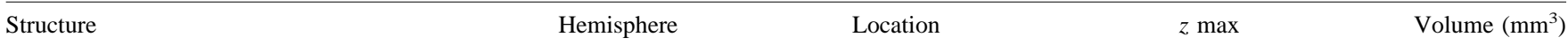

Experiment 1: BOLD time-course differences

Visual marking-related activation

SPL/Precun

$\mathrm{R}$

Post-search process-related activation

aIPS

pSTS

pSTS
$\mathrm{R}$

$\mathrm{L}$

$\mathrm{R}$

Experiment 2: BOLD amplitude differences

Visual marking-related activation

SPL/Precun

Claustrum/

$\mathrm{L}$

$\mathrm{L}$

Postsearch process-related activation

pSTS

$\begin{array}{rrr}7,-65,50 & 4.12 & 727 \\ 40,-45,40 & 3.47 & 124 \\ -44,-65,21 & 3.76 & 181 \\ 43,-53,19 & 4.20 & 446\end{array}$

727

124

446

Experiment 1: Visual marking-related time-course differences were characterized by early activation in the preview condition. Postsearch process-related time-course differences were characterized by late-activation onset in conjunction search. $Z$ values relate to timing differences (see text for details). Volume indicates the size of the activation. Coordinates indicate the locations of maximal differences according to the atlas by Talairach and Tournoux (1988).

Experiment 2: Visual marking-related activation was characterized by increased signal in the preview-only relative to the dummy-only condition. Postsearch process-related activation was characterized by increased activation in the single feature search-conjunction search contrast. $Z$ values relate to signal amplitude differences. Only activations with a volume $>=72 \mathrm{~mm}^{3}$ are listed.

L, left; R, right hemisphere; SPL, superior parietal lobule; aIPS, anterior intraparietal sulcus; pSTS, superior temporal sulcus, posterior segment; Precun precuneus.

with the onset of displays 1 or 2 , rather than shifts of the peak activation. Fig. 4 shows the BOLD time courses in right SPL in Experiment 1. As expected for a brain area involved in visual marking, the preview condition elicited an earlier onset of the BOLD response relative to the singlefeature search and conjunction search conditions, the activations of the latter two being almost indiscriminate. This impression was confirmed by an ANOVA over the time window from 0 to $4 \mathrm{~s}$ postonset of display 1 , which contains the initial part of the BOLD increase. In this time window, a significantly higher BOLD signal was observed in the preview condition relative to the single-feature and conjunction search conditions, as indicated by the significant main effects of condition (Table 3). Importantly, no significant

Table 2

Statistical analysis of BOLD time-course data

\begin{tabular}{|c|c|c|c|c|c|c|}
\hline \multirow[t]{2}{*}{ Time window: } & \multicolumn{3}{|l|}{$0-16 \mathrm{~s}$} & \multicolumn{3}{|l|}{$0-4 \mathrm{~s}$} \\
\hline & Condition & Time & Cond. $\times$ Time & Condition & Time & Cond. $\times$ Time \\
\hline \multicolumn{7}{|l|}{ Experiment 1} \\
\hline SPL $\quad \mathrm{R}$ & - & $* * *$ & $* *$ & $* *$ & $* * *$ & - \\
\hline aIPS $\quad \mathrm{R}$ & - & $* * *$ & $* *$ & - & $* * *$ & - \\
\hline pSTS L & - & $* *$ & - & $*$ & $* *$ & - \\
\hline pSTS R & - & $* * *$ & $* * *$ & - & $* *$ & - \\
\hline \multicolumn{7}{|l|}{ Experiment 2} \\
\hline SPL $\quad$ L & - & $* * *$ & $*$ & - & - & - \\
\hline SPL $\quad R$ & $* *$ & - & - & - & - & - \\
\hline pSTS R & - & $* * *$ & $* * *$ & $* *$ & $*$ & $*$ \\
\hline \multirow[t]{2}{*}{ Time window: } & \multicolumn{3}{|l|}{$0-8 \mathrm{~s}$} & \multicolumn{3}{|l|}{$8-16 \mathrm{~s}$} \\
\hline & Condition & Time & Cond. $\times$ Time & Condition & Time & Cond. $\times$ Time \\
\hline SPL L & $* *$ & $* * *$ & - & - & - & - \\
\hline
\end{tabular}


Table 3

Statistical analysis of BOLD time-course data

\begin{tabular}{|c|c|c|c|}
\hline Preview/conjunction search & Condition & Time & Cond. $\times$ Time \\
\hline \multicolumn{4}{|l|}{ Experiment 1} \\
\hline SPL $\quad \mathrm{R}$ & $*$ & $* * *$ & - \\
\hline aIPS $\mathrm{R}$ & - & $* *$ & - \\
\hline pSTS L & - & - & $*$ \\
\hline pSTS R & - & - & $*$ \\
\hline \multicolumn{4}{|l|}{ Experiment 2} \\
\hline pSTS R & - & $*$ & - \\
\hline \multicolumn{4}{|l|}{ Single feature/preview search } \\
\hline \multicolumn{4}{|l|}{ Experiment 1} \\
\hline SPL $\quad \mathrm{R}$ & $*$ & $* * *$ & $*$ \\
\hline aIPS $\mathrm{R}$ & - & $* * *$ & - \\
\hline pSTS L & - & - & - \\
\hline pSTS R & - & $* *$ & - \\
\hline \multicolumn{4}{|l|}{ Experiment 2} \\
\hline pSTS R & $*$ & $* *$ & - \\
\hline \multicolumn{4}{|c|}{ Single feature/conjunction search } \\
\hline \multicolumn{4}{|l|}{ Experiment 1} \\
\hline SPL $\quad \mathrm{R}$ & - & $* * *$ & - \\
\hline aIPS $\mathrm{R}$ & $*$ & $* * *$ & - \\
\hline pSTS L & $*$ & - & $* *$ \\
\hline pSTS R & $* *$ & $* *$ & - \\
\hline \multicolumn{4}{|l|}{ Experiment 2} \\
\hline pSTS R & $* *$ & - & $*$ \\
\hline
\end{tabular}

Comparisons between preview and single-feature search, preview and conjunction search, and single-feature and conjunction search in the onset time window $(0-4 \mathrm{~s})$. Levels of significance are indicated by $*(P<0.05)$, $* *(P<0.01)$, and $* * *(P<0.001)$.

difference was observed between single-feature and conjunction searches.

In right aIPS and pSTS, the overall ANOVA (0-16 s) yielded significant interactions of condition $\times$ time over the entire BOLD response, and in left pSTS a trend toward an interaction was observed $(P=0.093)$. The condition main effects were not significant. In the time window from 0 to $4 \mathrm{~s}$ after onset of display 2 a significant main effect was observed for conjunction search compared to single-feature search in all three areas. In the same time window, there was no significant difference in the comparison of preview and single-feature search in these areas, in line with our predictions. Contrary to expectation, there was also no significant main effect in the comparison of preview and conjunction search in any of the three areas, but in both left and right pSTS a significant interaction of condition $\times$ time was observed, reflecting the different slopes of the BOLD-signal (upward for preview, downward for conjunction search) within the time window (Fig. 4).

\section{Experiment 2: separation of activations elicited in the preview and search periods}

Activation elicited in the preview period. The preview-only conditions in Experiment 2 enabled us to examine activation to preview displays isolated from activation associated with search displays. Accordingly we contrasted green $\mathrm{H}$ preview trials with red $\mathrm{O}$ ("dummy") previews. This contrast yielded a significant signal increase in the left SPL/precuneus, at a virtually identical location (although in the contralateral hemisphere) to that associated with marking (in the preview condition) in Experiment 1 (Fig. 3b, Table 1). In the global ANOVA (time window 0-16 s), the condition main effect was not significant, but there was a significant interaction of condition (preview only, dummy only) $\times$ time. Fig. 4 shows that the preview-only trials elicited a higher initial activation compared to the dummy-only trials. Later on, both kinds of trials elicited a sustained increase of the BOLD response, with no apparent difference between the conditions. Therefore, we carried out additional analyses on the first half $(0-8 \mathrm{~s})$ and the second half $(8-16 \mathrm{~s})$ of the event-related response, to separate amplitude differences elicited from processing the preview or dummy display from subsequent amplitude differences which may arise due to differential expectations in the remainder of the trial (which contained no search display). There was a significant condition main effect in the first half, confirming the increased activation elicited by the preview display. No significant main effect or interaction was observed in the second half, yielding no indication of differential activity elicited by the two conditions after the offset of the preview display. A slightly earlier onset in the preview condition was indicated by a marginally significant condition main effect $(P=0.051)$ in the time window from 0 to $4 \mathrm{~s}$ after onset of the preview display.

It was puzzling to find a marking-associated activation pattern in left SPL in Experiment 2, whereas we had found a marking-related pattern in right SPL, at the mirror image location, in Experiment 1. In order to clarify the role of the right SPL in visual marking, we carried out an ROI analysis at the location of the activation maximum in Experiment 1. We found an activation pattern qualitatively comparable to that observed in left SPL, with somewhat increased activation in the preview-only condition compared to the dummyonly condition. However, the time courses were much noisier than in left SPL, and the time course analyses yielded no significant effects.

Another significant activation was observed in the left putamen.

Activation elicited in the search period. It may be objected ${ }^{2}$ that the activation accompanying the preview displays is not specific for visual marking, but reflects general attentional processing of the meaningful preview stimuli, which is not present with the dummy stimuli. If this were true, we should observe similar activation when contrasting the conjunction with the feature search displays, because conjunction search requires the attentive processing of the search stimuli,

\footnotetext{
${ }^{2}$ As pointed out by one reviewer.
} 
whereas feature search is mostly preattentive. We thus contrasted display 2 of the conjunction search condition with display 2 of the feature search condition. We also lowered the significance threshold to $\alpha=0.005$ in order to capture potential near-threshold activations. However, no significant activation was observed in the SPL or precuneus of the left or right hemisphere. Moreover, we looked whether significant activation could be observed at the exact locations of marking-related SPL activations in Experiments 1 and 2 at the $\alpha=0.05$ level. This was not the case. These analyses underline the specificity of the marking-related activation pattern in SPL.

We have interpreted the early onset of activation in the pSTS and aIPS in Experiment 1 in the single-feature and preview conditions as an indicator of target detection or response preparatory processes. To test this hypothesis in Experiment 2, we analyzed differences in amplitude elicited during presentation of display 2 , with confounds due to display 1 processing removed (see Methods for details). Targets were most salient in the single-feature search condition of Experiment 2. Subjects detected targets significantly faster in single-feature search, compared to conjunction search. Moreover, with a search slope of $10 \mathrm{~s}$ per item, single-feature search was the only condition eliciting target pop-out. Therefore, we expected display 2-related activation to be higher in single feature search compared to conjunction search. This pattern was indeed found in the right pSTS area, at the same location at which we detected a delayed response in conjunction search in Experiment 1 (Fig. 3). While response times were also significantly faster in the preview condition, compared to conjunction search, the slope observed in the preview condition lay in between the slopes of the single-feature search and conjunction search conditions. Likewise, the BOLD response elicited by the preview condition lay in between the responses elicited by single-feature and conjunction search, both in terms of amplitude and latency (Fig. 4). Statistical analysis of the time courses yielded a nonsignificant overall (time window 0-16 s) condition main effect, but a significant interaction of condition $\times$ time. In the time window 0 - to 4 -s post display 2 onset, there were significant differences between the conditions, which broke down into significantly increased signal in this onset phase for single-feature search compared to conjunction search and preview search, with a tendency for an increased response (indicating earlier onset) for preview relative to conjunction search $(P=0.068$; Table 3$)$.

\section{Discussion}

In Experiment 1 we used the information inherent in the time course of activation to differentiate between (i) visual marking of old distractors, which was associated with the superior parietal lobule in the preview condition, and (ii) target detection and/or response-preparatory processes, which were associated with activation in the inferior parietal lobule. These basic results were replicated in Experiment 2 where we used a contrasting procedure, based on measured differences in the magnitude of the BOLD response. Here visual marking was assessed using preview-only trials, when search displays were not presented. In both experiments we used two baseline conditions, with easy (singlefeature) and difficult (conjunction) search. The latter resulted in significantly longer search times. In the critical preview condition, one set of distractors from the conjunction search display was presented immediately before the actual search display. In the other search conditions, dummy items (not future distractors) were presented in display 1 . In accordance with previous behavioral studies, the early presentation of distractor items (in the preview condition) led to fast search times, relative to conjunction search. In Experiment 1 the slopes for the preview condition matched those in the single-feature (easy) baseline. In Experiment 2 overall RTs in the preview and single-feature baseline did not differ.

Previous behavioral results have indicated that this beneficial effect of the preview display is contributed to by an active bias against the advance distractors, a process we have termed visual marking (Watson and Humphreys, 1997, 1998, 2000). In search of the neural structures involved in this process in Experiment 1, we looked for brain areas which would show a particular temporal activation pattern: earlier onset of activation in the preview condition relative to the other search conditions. Early activation for the preview condition was observed in right posterior SPL. BOLD timecourse analysis confirmed that the contrasting time courses were not contaminated by BOLD amplitude differences. The behavioral evidence indicates that a process of visual marking involving the advance encoding and rejection of old distractors enables them to be ignored. The imaging data suggest that this process is supported by the posterior SPL at the border to the precuneus. In a second experiment, we analyzed differences in the magnitude of activation elicited by the preview (display 1) and subsequent search displays (display 2) separately. We confirmed the role of SPL/precuneus in visual marking in that the advance information in the preview condition was associated with a signal increase in the exact location as in Experiment 1, although now in the left hemisphere. We cannot determine, based on our data, why there was a shift from a right marking-related activation pattern in Experiment 1 to a left-dominant activation pattern in Experiment 2. A possible cause may be the change in the stimuli from Experiment 1 to Experiment 2, which may have produced a narrowing of the focus of attention, to the center items, in Experiment 2. This narrowing may not have occurred in Experiment 1 because there was not then a constant set of stimuli in the periphery that had to be ignored (the border items, used in Experiment 2). Nevertheless, the exact overlap in the coordinates in the SPL, across the two experiments, is noteworthy.

Since visual marking involves both the encoding and the rejection of old distractors (Humphreys et al., 2002), it is 
difficult to assess which component is mediated by activation in the SPL. However, we failed to find any increase in activation in this area in a comparison of the single-feature and conjunction conditions, even though attentional involvement in search is greater in the conjunction condition. This suggests that the increased SPL activation was not simply due to greater attention to the first display in the preview condition, but specifically reflects processes involved in visual marking.

In a study of general attentional mechanisms in posterior parietal cortex, Wojciulik and Kanwisher (1998) found common activation across different forms of visual selection, including spatial shifts of attention, matching of spatially distinct objects, and nonspatial feature conjunction discrimination (in rapid serial visual presentation) at locations $(x=-15, y=-64, z=48 ; x=14, y=-68, z=$ 48) almost identical to the locations which we found associated with visual marking in the present study $(x=-7, y$ $=-62, z=52 ; x=7, y=-65, z=50$ ). They proposed that posterior SPL may be involved in the suppression of irrelevant distractors. They noted that previous imaging experiments led to posterior parietal activation when the task contained irrelevant distractors, and that parietal activation was absent in those studies of visual attention which did not contain distractor elements which had to be inhibited in order to carry out the task (e.g., when single-conjunction or feature-defined targets have to be categorized; cf. Rees et al., 1997). Our data are in good agreement with this hypothesis, in that they indicate that the SPL/precuneus is specifically involved in visual marking and efficient rejection of old distractors in search.

Inhibitory processes in visual marking have been demonstrated behaviorally by the finding that luminance onsets at the locations of old (previewed) distractors are difficult to detect (Olivers and Humphreys, 2002; Watson and Humphreys, 2000). Furthermore, with static displays, there is evidence of inhibition applied to the locations of the old items (Olivers et al., 1999). This suggests that visual marking can be based on the inhibition of spatial locations. Our finding, that early posterior SPL activation was specific to the condition where visual marking occurred, would fit with this brain area being the site of a spatial map, which is constructed for the preview display, and which serves as the locus of spatial inhibition. However, from the present results we cannot distinguish whether the activation reflects a spatial map constructed for the old items or inhibition applied to that map.

In support of a role of the right SPL in spatial selection mechanisms, Coull and Frith (1998) found that the right posterior SPL (close to our marking-related activation) was specifically activated by tasks requiring spatial selection, whereas the right IPS was active in tasks with nonspatial as well as spatial selection. Also, spatial attention led to more medial activation in the SPL than nonspatial attention in the experiments of Wojciulik and Kanwisher (1998). Furthermore, imaging studies of spatial attention often show pos- terior SPL activation in addition to IPS activation (Gitelman et al., 1999; Hopfinger et al., 2000).

It should also be noted that it is unlikely that the SPL activation we observed, with early onset in the preview condition, was due to eye movements made to the old distractor items. First, observers were asked to remain fixated during the preview period. Second, as we have noted, there was no difference between the single-feature and the conjunction conditions within this region. Third, and more telling, Olivers et al. (2002) have shown that search performance is actually rather poor when participants first make a serial scan of the items in a preview display before a search display is presented. They had participants search for a target that could be in the preview or the search display. After searching the preview, participants were allowed to prepare themselves and then press a button to onset the search display. Despite this, performance was only a little better than in a baseline in which the items from the preview and search displays all appeared simultaneous (equivalent to the conjunction baseline here). The detection of targets in search displays was much more efficient in a marking condition in which participants did not search previews but were instead set against previews from their onset (knowing the target could never appear in the preview displays). The shallower search slopes observed in the preview relative to the conjunction condition here provide behavioral evidence against the SPL activity reflecting a serial search of previews.

In contrast to the marking-related activity in the SPL, the right aIPS and the bilateral pSTS areas showed no early onset of activation related to the processing of advance distractors (in the preview condition), but a delayed onset of the evoked response in difficult (conjunction) search relative to easy (single-feature and preview) search conditions. This pattern was expected in areas which are involved in postsearch processes (target detection and/or response preparation), which are delayed in conjunction search. In Experiment 2, we replicated the delayed onset of activation in conjunction search, relative to single-feature search, in the right pSTS, at the same location as in Experiment 1. Comparison of signal time courses showed a very similar pattern in both experiments. In both experiments, this pattern was more clear-cut in right pSTS. However, the data from Experiment 1 suggest that the difference between left and right pSTS is a difference in the degree of involvement, rather than a categorical processing difference.

Lesions of the right temporoparietal junction area (including the pSTS) have a strong association with contralateral neglect (Vallar, 1993). Lesions of the TPJ area, but not more superior parietal lesions, led to deficient target detection in the contralesional visual hemifield, especially when the patients were miscued to the ipsilesional hemifield (Friedrich et al., 1998). A recent event-related fMRI study reported activations in the TPJ region specifically associated with target processing (most strongly when the target appeared at an unexpected location, but not with processing of 
cues which preceded the targets; Corbetta et al., 2000). Thus there is converging evidence from clinical and neuroimaging studies that the TPJ region is vital for target detection.

Postsearch, specifically response-related, time-course patterns bordering the ascending limb of the IPS are consistent with impaired coordination of finger movements after a lesion in this area (Binkofski et al., 1998) as well as fMRI activations along the ascending and horizontal limbs of the IPS when prehensile hand movements are made (Binkofski et al., 1998, 1999). These activations have in common that they are all more lateral (and mostly more anterior) than the marking-related SPL activations in our study but included the present postsearch activation at the lateral bank of the ascending IPS. While conjunction search elicited a delayed response in both Experiments in right pSTS, we did not find a postsearch activation pattern in aIPS in Experiment 2. A possible explanation may be that the randomization of search conditions in Experiment 2 minimized differences in response preparation whereas the blocked presentation in Experiment 1 may have led to differences in the readiness to respond depending on the type of search, with high readiness in easy search and lower readiness in conjunction search, in which responses could only be elicited after a protracted search. AIPS neurons may also respond to visual object shape, independent of grasping (Murata et al., 2000). In our study, this may imply that aIPS is involved in the chain of processes betwen detection of the target and eliciting of the proper response. Clearly, further research is needed to investigate this issue.

In conclusion, our data suggest that distinct areas in posterior parietal cortex support distinct processing steps in visual search and attention. The SPL supports selection biases against old stimuli to benefit the selection of new objects. The pSTS area, in contrast, subserves target detection, which is followed by response programming in the cortex on the lateral bank of the ascending limb of the IPS.

\section{Acknowledgments}

This study was supported by grants from the Deutsche Forschungsgemeinschaft (DFG Working Memory Research Group Grant SCHR 375/8-1) and the Medical Research Council (UK, Grant G9623474). We thank two anonymous reviewers for their helpful comments.

\section{References}

Binkofski, F., Buccino, G., Posse, S., Seitz, R.J., Rizzolatti, G., Freund, H.-J., 1999. A fronto-parietal circuit for object manipulation in man: evidence from an fMRI study. Eur. J. Neurosci. 11, 3276-3286.

Binkofski, F., Dohle, C., Posse, S., Stephan, K.M., Hefter, H., Seitz, R.J., Freund, H.-J., 1998. Human anterior intraparietal area subserves prehension. A combined lesion and functional MRI activation study. Neurology 50, 1253-1259.

Bosch, V., 2000. Statistical analysis of multi-subject fMRI data: the assessment of focal activations. J. Magn. Reson. 11, 61-64.
Corbetta, M., Kincade, J.M., Ollinger, J.M., McAvoy, M.P., Shulman, G.L., 2000. Voluntary orienting is dissociated from target detection in human posterior parietal cortex. Nature Neurosci. 3, 292-297.

Coull, J.T., Frith, C.D., 1998. Differential activation of right superior parietal cortex and intraparietal sulcus by spatial and nonspatial attention. NeuroImage 8, 176-187.

Dymond, R., Norris, D.G., Pollmann, S., Zysset, S., 1999. Application of double voxel functional spectroscopy to event-related cognitive experiments. Magn. Reson. Med. 41, 217-223.

Friedrich, F.J., Egly, R., Rafal, R.D., Beck, D., 1998. Spatial attention deficits in humans: a comparison of superior parietal and temporalparietal junction lesions. Neuropsychology 12, 193-207.

Friston, K.J., Fletcher, P., Josephs, O., Holmes, A., Rugg, M.D., Turner, R., 1998. Event-related fMRI: Characterizing differential responses. NeuroImage 7, 30-40.

Friston, K.J., Frith, C.D., Turner, R., Frackowiak, R.S.J., 1995. Characterizing evoked hemodynamics with fMRI. NeuroImage 2, 157-165.

Friston, K.J., Holmes, A.P., Worsley, K.J., Poline, J.B., Frith, C.D., Frackowiak, R.S.J., 1995. Statistical parametric maps in functional imaging: A genral linear approach. Hum. Brain Mapp. 2, 189-210.

Friston, K.J., Williams, S., Howard, R., Frackowiak, R.S.J., Turner, R., 1996. Movement-related effects in fMRI time-series. Magn. Reson. Med. 35, 346-355.

Gitelman, D.R., Nobre, A.C., Parrish, T.B., LaBar, K.S., Kim, Y.-H., Meyer, J.R., Mesulam, M.M., 1999. A large-scale distributed network for covert spatial attention. Brain 122, 1093-1106.

Henson, R.N.A., Price, C., Rugg, M.D., Turner, R., Friston, K., 2002. Detecting latency differences in event-related BOLD responses: application to words versus nonwords, and initial versus repeated face presentations. NeuroImage 15, 83-97.

Hopfinger, J.B., Buonocore, M.H., Mangun, G.R., 2000. The neural mechanisms of top-down attentional control. Nature Neurosci. 3, 284-291.

Humphreys, G.W., Watson, D.G., Joliceour, P., 2002. Fractionating visual marking: dual task decomposition of the marking state by timing and modality. J. Exp. Psychol. Hum. Percept. Perform. 28, 640-660.

Josephs, O., Henson, R.N.A., 1999. Event-related functional magnetic resonance imaging: modelling, inference and optimization. Philos. Trans. R. Soc. London B 354, 1215-1228.

Josephs, O., Turner, R., Friston, K., 1997. Event-related fMRI. Hum. Brain Mapp. 5, 243-248.

Lohmann, G., Mueller, K., Bosch, V., Mentzel, H., Hessler, S., Chen, L., Zysset, S., von Cramon, D.Y., 2001. Lipsia-A new software system for the evaluation of functional magnetic resonance images of the human brain. Comput. Med. Imaging Graphics 25, 449-457.

Menon, R., Luknowsky, D., Gati, J., 1998. Mental chronometry using latency-resolved functional MRI. Proc. Natl. Acad. Sci. USA 9518, 10902-10907.

Murata, A., Gallese, V., Luppino, G., Kaseda, M., and Sakata, H, 2000. Selectivity for the shape, size, and orientation of objects for grasping in neurons of monkey parietal area AIP. J. Neurophysiol. 83, 2580-2601.

Oldfield, R.C., 1971. The assessment and analysis of handedness: the Edinburgh inventory. Neuropsychologia 9, 97-113.

Olivers, C.N.L., Humphreys, G.W., 2002. When visual marking meets the attentional blink: more evidence for top-down limited-capacity inhibition. J. Exp. Psychol. Hum. Percept. Perform 28, 22-42.

Olivers, C.N.L., Humphreys, G.W., Heinke, D., Cooper, A.C.G., 2002. Prioritization in visual search: visual marking is not dependent on a mnemomic search. Percept. Psychophys. 64, 540-560.

Olivers, C.N.L., Watson, D.G., Humphreys, G.W., 1999. Visual marking of locations and feature maps: evidence from within dimension defined conjunctions. Q. J. Exp. Psychol. 52A, 679-715.

Pollmann, S., Wiggins, C.J., Norris, D.G., von Cramon, D.Y., Schubert, T., 1998. Use of intertrial intervals in single-trial experiments: a 3T fMRI study. NeuroImage 8, 327-339.

Rees, G., Frackowiak, R., Frith, C., 1997. Two modulatory effects of attention that mediate object categorization in human cortex. Science $275,835-838$. 
Talairach, J., Tournoux, P., 1988. Co-planar sterotactic atlas of the human brain. Thieme, Stuttgart.

Theeuwes, J., 1994. Stimulus-driven capture and attentional set: selective search for color and visual abrupt onsets. J. Exp. Psychol. Hum. Percept. Perform. 20, 799-806.

Theeuwes, J., Kramer, A.F., Atchley, P., 1998. Visual marking of old objects. Psychol. Bull. Rev. 5, 130-134.

Treisman, A., Gelade, G., 1980. A feature-integration theory of attention. Cogn. Psychol. 12, 97-136.

Treisman, A., Gormican, S., 1988. Feature analysis in early vision: evidence from search asymmetries. Psychol. Rev. 95, 15-48.

Vallar, G., 1993. The anatomical basis of spatial hemineglect in humans, in: Robertson, I.H., Marshall, J.C (Eds.), Unilateral Neglect: Clinical and Experimental Studies. Hove, LEA, pp. 27-62.
Watson, D.G., Humphreys, G.W., 1998. Visual marking of moving objects: a role for top-down feature based inhibition in selection. J. Exp. Psychol. Hum. Percept. Perform. 24, 946-962.

Watson, D.G., Humphreys, G.W., 2000. Visual marking: evidence for inhibition using a probe-dot detection paradigm. Percept. Psychophys $62,471-481$.

Watson, D.G., Humphreys, G.W., 1997. Visual marking: prioritizing selection for new objects by top-down attentional inhibition of old objects. Psychol. Rev. 104, 90-122.

Wojciulik, E., Kanwisher, N., 1998. The generality of parietal involvement in visual attention. Neuron 23, 747-764.

Yantis, S., 1996. Attentional capture in vision, in: Kramer, A.F., Coles, M., Logan, G. (Eds.), Converging Operations in the Study of Visual Selective Attention. Am. Psychol. Assoc., Washington, DC, pp. 45-76. 